\title{
Anti-angiogenic Properties of Bevacizumab Improve Respiratory System Inflammation in Ovalbumin-Induced Rat Model of Asthma
}

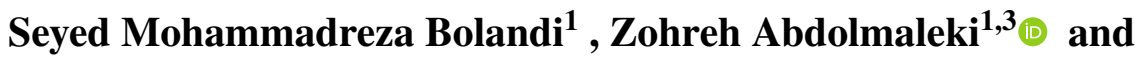 \\ Mohammad-Ali Assarehzadegan ${ }^{2}$
}

Received 20 June 2020; accepted 7 July 2021

Abstract-Studies on the bronchial vascular bed have revealed that the number of blood vessels in the lamina propria and under the mucosa of the lung tissue increases in patients suffering from mild to severe asthma. Thus, in this study, a new strategy was employed in respiratory system disorders by angiogenesis inhibition in an ovalbumin (OVA)-induced rat model of asthma. Twenty-one male Wistar albino rats, 8 weeks old, were randomly divided into three groups ( $n=7$ in each group), including (1) control group, (2) OVAtreated group, and (3) OVA + Bmab (bevacizumab drug). On days 1 and 8, $1 \mathrm{mg}$ of OVA and aluminum hydroxide in sterile phosphate-buffered saline (PBS) were intraperitoneally injected to rats in groups 2 and 3 . The control group was only subject to intraperitoneal injection of saline on days 1 and 8. One week after the last injection, the rats (groups 2 and 3) were exposed to OVA inhalation for $30 \mathrm{~min}$ at 2-day intervals from days 15 to 25. After sensitization and challenge with OVA, the OVA + Bmab group (group 3) were treated with a $5 \mathrm{mg} / \mathrm{kg}$ bevacizumab drug. Genes and protein expression of IL- $1 \beta$ and TNF- $\alpha$ and the expression of vascular endothelial growth factor (VEGF) protein were assessed by real-time PCR and immunohistochemistry respectively, in lung tissue. OVA exposure increased mucosal secretion and inflammatory cell populations in lung tissue and OVAspecific IgE level in serum. Also, VEGF and cytokine factor expression were significantly elevated in the OVA-induced asthma model $(p \leq 0.05)$. However, rats in OVA + Bmab group showed significantly a decrease in VEGF and IL- $1 \beta$ and TNF- $\alpha$ genes as well as proteins $(p \leq 0.05)$. The results showed that bevacizumab efficiently diminished bronchial inflammation via downregulation of VEGF expression, followed by inflammatory cells population and cytokines reduction. Angiogenesis inhibition in rats with induced asthma not only suppresses the inflammatory process through blocking VEGF expression but also inhibits the development of new blood vessels and progressing asthmatic attacks.

KEY WORDS angiogenesis inhibitors; asthma; cytokines; bevacizumab drug; ovalbumin.

${ }^{1}$ Department of Pharmacology, Karaj Branch, Islamic Azad University, Karaj, Iran

${ }^{2}$ Immunology Research Center, Institute of Immunology and Infectious Diseases, Iran University of Medical Sciences, Tehran, Iran

${ }^{3}$ To whom correspondence should be addressed at Department of Pharmacology, Karaj Branch, Islamic Azad University, Karaj, Iran Email: zohreh.abdolmaleki@kiau.ac.ir

\section{INTRODUCTION}

Respiratory disease, which is commonly associated with lung disease, includes a group of diseases that interferes with the function of the lungs by involving different parts of the respiratory tract. Respiratory disease can 
sometimes be caused by damage to the pleural membrane, the pleural cavity, or the muscles and nerves of the respiratory tract. Lung disease may involve the airways, lung tissues, or blood vessels $[1,2]$. A combination of these disorders is commonly seen in many respiratory illnesses. In general, respiratory diseases can be divided into the following groups: pulmonary obstructive pulmonary disease [3], lung restrictive diseases [4], pulmonary vascular disease [5], and other diseases. Asthma is a very common disease that involves a complex interaction of environmental factors, airflow obstruction, high bronchial blood pressure, and inflammation [6,7]. The most significant feature of clinical symptoms is the contraction of smooth muscles and inflammation, which result in the narrowing of the airways and obstruction. Bronchial obstruction, including respiratory infections, allergic reactions, irritants, exercise, and non-steroidal anti-inflammatory drugs can occur by countless stimuli [8].

Persistent inflammation in the airways can lead to structural changes, including mucosal prolapse, smooth muscle hyperplasia, subepithelial fibrosis, proliferation of blood vessels, and infiltration of inflammatory cells. Currently, several mechanisms implicated in airway inflammation are presumed to be involved in bronchial microcirculation changes in asthma [9]. The changes are vasodilation, increased blood flow, angiogenesis, and increased vascular permeability. This structural change occurs usually in inflammation, yet little is known about the functional significance of bronchial vascular remodeling in asthma [10]. Anti-angiogenesis therapy represents one of the most significant advances in clinical oncology [11]. Bevacizumab is known as a monoclonal antibody against vascular endothelial growth factor (VEGF) [12]. Therefore, VEGF is a basic angiogenic factor involved in both normal physiological processes, such as embryonic development and wound healing, and diseases such as cancer. Bevacizumab is a recombinant humanized full-length monoclonal antibody with six VEGF-binding residues that bind to VEGF, preventing VEGF from binding to its target, namely, VEGFR-1 and VEGFR-2, on endothelial cells that obstruct angiogenesis by blocking VEGF isoforms. However, it was initially used to treat metastatic colorectal cancer intravenously. The efficiency profile of bevacizumab is comparable to ranibizumab, but its economic usefulness is more being a cost-effective replacement [13, 14]. Bevacizumab binds to VEGF ligands and decreases tumor angiogenesis; it can down the rate of tumor growth, thereby improving quality of life and survival for patients suffering from cancer [15]. It efficiently diminishes bronchial inflammation by reducing the expression of VEGFR2 and anti-inflammatory cytokine (IL-10). Regarding the effectiveness of bevacizumab, it seems that the clinical therapeutic usefulness of anti-IL-5 or anti-IL-5-producing cells in asthma/allergy treatment remains to be an intriguing possibility[16]. Nearly four decades ago, the strategy of stopping tumor growth and metastasis by blocking tumor angiogenesis was proposed by Judah Folkman [17]. Anti-angiogenesis therapy inhibits tumor growth and restrains metastasis by cutting the fuel supply and destroying the circulating pathway for the tumor cells by blocking tumor angiogenesis [18]. Recent studies have demonstrated an increased airway mucosal blood flow by dilatation of resistance arteries and increased number of vessels in airways of asthma [19-21]. In addition, most inflammatory mediators cause bronchial vasodilation in animal models [22-26]. It is also postulated that the increase in bronchial microcirculation and airway blood flow amplifies inflammatory responses by acting as a gateway to the subepithelium for inflammatory cells, although some reports have claimed that increased airway blood flow may play a role in removing inflammatory mediators from airways $[27,28]$. Moreover, the increase in the number and size of vessels can contribute to the thickening of the airway wall, which in turn may lead to critical narrowing of the bronchial lumen, as bronchial smooth muscle contraction occurs [28-30]. Hence, in the present study, the effect of bevacizumab on asthma was investigated in rat models through analyzing goblet cell population, leukocytes in bronchoalveolar lavage fluid (BALF), and cell migration focusing on angiogenesis marker, VEGF, and inflammatory cytokines IL- $1 \beta$ and TNF- $\alpha$.

\section{MATERIALS AND METHODS}

\section{Preparation of Animal Model and Study Groups}

Twenty-one male Wistar albino rats, 8 weeks old, weighing between 250 and $300 \mathrm{~g}$, were purchased from Pasteur Institute, Tehran, Iran. The animals were kept under controlled conditions at $22{ }^{\circ} \mathrm{C}$, with $45 \%$ humidity, 12-h light cycles, and free access to food, water, and libitum. The study procedures were performed according to the Ethical Committee of Azad University. The ethical code assigned for this study was IR.IAU.K.REC.1398.088. Ovalbumin grade V (OVA), aluminum hydroxide, and other compounds were purchased from Sigma Chemicals, St Louis, USA. All 
animals were randomly divided into three groups $(n=7$ in each group), including (1) control group, (2) OVAtreated group, and (3) OVA + Bmab (bevacizumab drug). Sensitization and challenge instructions relevant to working with rats were performed as previously described [31] (Fig. 1). Aluminum hydroxide solution (Aldrich, Milwaukee, WI) was diluted in saline buffer and incubated with OVA (grade V; Sigma, St. Louis, MO) overnight. On days 1 and $8,1 \mathrm{mg}$ of OVA and $200 \mathrm{mg}$ of aluminum hydroxide (Aldrich, Milwaukee, WI) in $0.5 \mathrm{~mL}$ sterile phosphate-buffered saline (PBS) were intraperitoneally injected into rats in groups 2 and 3 . The control group was only subject to intraperitoneal injection of saline on days 1 and 8. One week after the last injection, the rats (groups 2 and 3) were exposed to OVA inhalation for $30 \mathrm{~min}$ at 2-day intervals from days 15 to 25 .

\section{Drug Treatment}

After preparation of asthma rat models through OVA exposure protocols, the OVA + Bmab group (group 3) were treated with $2.5 \mathrm{mg} / \mathrm{kg}$ bevacizumab drug (Avastin, Roche $25 \mathrm{mg} / \mathrm{mL}$, Basel, Switzerland) diluted in $9 \%$ $\mathrm{NaCl}$ solution to a final concentration of $5 \mathrm{mg} / \mathrm{mL}$ [32]. Two days after the end of the study, the rats were euthanized with ketamine/xylazine and blood samples were collected, and their lung tissues were isolated for further examinations.

\section{Gene Expression Evaluation of $I L-1 \beta$ and $T N F-\alpha$}

The quantitative expression of $I L-1 \beta$ and $T N F-\alpha$ genes was evaluated in lung tissues obtained from all three study groups of rats by Real-Time PCR System (Applied Biosystems, Warrington, UK). To this end,
Table 1 The Primers Used to Detect $I L-1 \beta$ and $T N F-\alpha$ Expression

\begin{tabular}{ll}
\hline Gene & Primer sequence $\left(5^{\prime}-3^{\prime}\right)$ \\
\hline $\boldsymbol{I L}-\boldsymbol{1} \boldsymbol{\beta}-\mathrm{F}$ & TGTGACTGGTGGGATGATGA \\
$\boldsymbol{I L} \boldsymbol{- 1} \boldsymbol{\beta}-\mathrm{R}$ & GTTCTGTCTATTGAGGTGGAGA \\
$\boldsymbol{T} \boldsymbol{N} \boldsymbol{F} \boldsymbol{\alpha}-\mathbf{F}$ & GAGATGTGGAAATGGCAGAGGA \\
$\boldsymbol{T} \boldsymbol{N} \boldsymbol{F} \boldsymbol{\alpha}-\mathbf{R}$ & GAGAAGATGATGTGAGTGTGAGG \\
$\boldsymbol{G A P}-\mathbf{R}$ & CATACTCAGCACCAGCATCACC \\
$\boldsymbol{G A P}-\mathbf{F}$ & AAGTTCAACGGCACAGTC \\
& AAGG \\
\hline
\end{tabular}

total RNA from lung tissues was extracted by TRIzol reagent (Invitrogen, Thermo Fisher Scientific, Inc., Waltham, MA, USA) according to the manufacturer's protocols, and subject to RNase-free DNase treatment. Then, RNA samples were transcribed by using a reverse transcriptase enzyme, and cDNAs were applied to qPCR. The primers designed for detecting $I L-1 \beta$ and $T N F$ - $\alpha$ gene expression were represented in Table 1. The real-time PCR program employed in all processes was defined as follows: $2 \mathrm{~min}$ at $50{ }^{\circ} \mathrm{C}$ and $10 \mathrm{~min}$ at $95^{\circ} \mathrm{C}$, followed by 40 two-step cycles comprising of $95^{\circ} \mathrm{C}$ for $15 \mathrm{~s}$ and $60{ }^{\circ} \mathrm{C}$ for $60 \mathrm{~s}$. Finally, the relative gene expression was examined through the $2-\Delta \Delta \mathrm{Ct}$ method. Each assay was run in triplicate.

\section{Immunohistochemistry of IL-1B, TNF- $\alpha$, and VEGF}

The lung tissues obtained from all three study groups of rats were steeped with buffered formalin in PBS, pH 7.2, and then infused in formalin at $4{ }^{\circ} \mathrm{C}$ overnight. After the fixation period, the lung tissues were embedded in paraffin. Six-micrometer sections of tissues

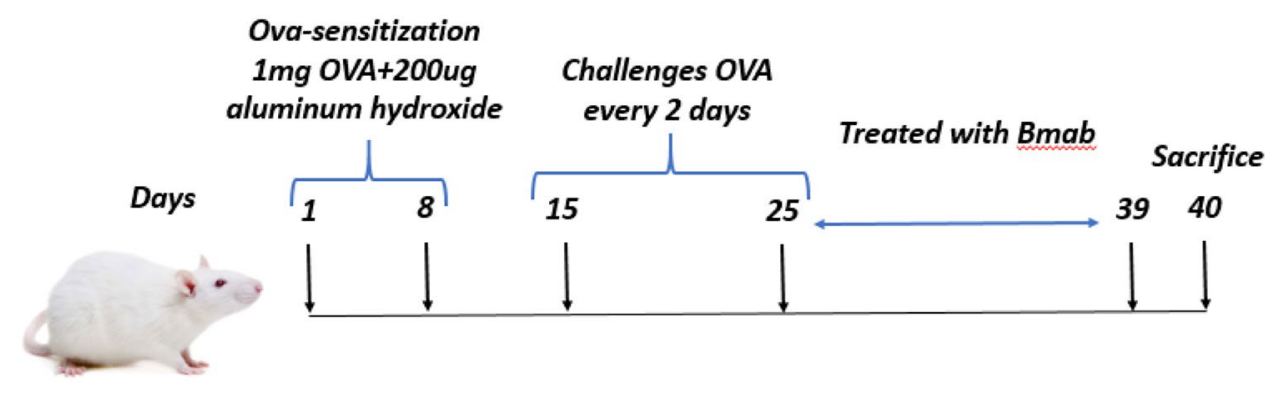

Fig. 1 Effects of Bmab on OVA-induced asthmatic rats. The schematic protocol of the study. Bmab, Bevacizumab; OVA, ovalbumin. 
were prepared, deparaffinized, and dehydrated. The samples were incubated in $50 \%$ formamide solution and $2 \times$ standard sodium citrate buffer at $65^{\circ} \mathrm{C}$ for $2 \mathrm{~h}$ and then incubated twice in $100 \mathrm{mM}$ of sodium borate $(\mathrm{pH}=8.5)$. The samples were blocked with $0.4 \%$ Triton $\mathrm{X}-100$ in PBS and $10 \%$ goat serum for $30 \mathrm{~min}$. Next, the sections were incubated with monoclonal primary antibodies in PBS containing $0.05 \%$ Tween- 20 and $1 \%$ BSA at $4{ }^{\circ} \mathrm{C}$ overnight. The five used primary antibodies were against IL-1 $\beta$ (rabbit anti-IL-1 $\beta ; 1: 100$, Abcam, Cambridge, UK), TNF- $\alpha$ (rabbit anti-TNF- $\alpha ; 1: 100$, Abcam, Cambridge, UK), and VEGF (Rabbit anti-VEGF; 1:100, Abcam, Cambridge, UK). Afterward, the samples were washed with PBS and exposed to FITC-conjugated secondary antibodies (goat anti-rabbit; 1:200, Abcam, Cambridge, UK) diluted in the PBS at $37^{\circ} \mathrm{C}$ for $90 \mathrm{~min}$ in the dark. DAPI was used for nuclear staining. The samples were visualized under a light microscope (Olympus, Japan) at $400 \times$ magnification. FITC expression was measured by ImageJ software based on nuclear staining, indicating a positive reaction for the desired markers.

\section{Periodic Acid-Schiff Staining and Goblet Cell Population}

To detect the histopathological changes indicating inflammation such as the hyperplasia goblet cells in lung tissues affected by OVA treatment, the PAS staining was used on formalin-fixed tissue sections. In brief, the coverslips containing the hydrated tissue sections were immersed in $0.5 \%$ periodic acid solution for $2 \mathrm{~min}$ and then rinsed in tap water for $5 \mathrm{~min}$. Next, the coverslips were placed in Schiff reagent for $30 \mathrm{~min}$ and again washed with tap water for $15 \mathrm{~min}$. To differentiate the diagnosis, the tissues were stained with Harris hematoxylin solution (Sigma-Aldrich, Germany) for 6 min and washed with tap water for $5 \mathrm{~min}$. Finally, the tissues were dehydrated and mounted using a mounting medium. The samples were examined under a light microscope (Olympus, Japan) at $100 \times$ and $400 \times$ magnification, and the PAS-positive goblet cells were counted measured by ImageJ software based on nuclear staining.

\section{Differential Cell Counting in BALF}

To evaluate the inflammatory cell population, the lung tissues were removed from the sacrificed animals and prepared for differential cell count as described by
Yang et al. [33]. Briefly, left lungs were washed thrice with $1 \mathrm{ml}$ saline, and the lavages were pooled (mean volume, $2.0 \pm 0 \mathrm{ml}$ ). The retrieved volume of BAL was centrifuged immediately at $3000 \mathrm{rpm}$ for $10 \mathrm{~min}$ at $4{ }^{\circ} \mathrm{C}$. Then, cell pellets were resuspended and washed twice in PBS. The differential cell count was performed with Wright-Giemsa staining by two operators blind to the groups. The results are expressed as the total cell number $\times 10^{5}$.

\section{Measurement of Serum OVA-Specific IgE}

To analyze the level of OVA-specific IgE in serum, blood samples were collected from rats by cardiac puncture. Whole blood was centrifuged and the serum samples were collected. VA-specific IgE was determined by an enzyme-linked immunosorbent assay (ELISA) kit based on manufacturer protocol (R\&D Systems, Inc., Minneapolis, MN, USA). Absorbance was measured at $450 \mathrm{~nm}$ using a plate reader (BioTek ELx800, USA).

\section{Statistical Analysis}

There were 7 animals $(n=7)$ per group, and specimens were taken from all 21 animals for each assay. All experiments, including real-time PCR, IHC, PAS, cell counting, and ELISA, were conducted at least 3 times. The analysis of the resulting data was performed using GraphPad Prism version 6.0. The results were represented as the mean \pm standard deviation. Statistical differences were analyzed using one-way ANOVA and Tukey post hoc test. The $p<0.05$ was considered significant.

\section{RESULTS}

\section{Goblet Cell Population}

Goblet cell population decreased significantly in airways after treatment with bevacizumab compared to the asthma group $(p<0.05)$. Meanwhile, goblet cell hyperplasia was observed in rats treated with OVA in the asthmatic model (Fig. 2 A and B).

\section{Gene Expression of Cytokines in Lung Tissue}

The gene expression of $I L-1 \beta$ and TNF- $\alpha$ cytokines was evaluated in three animal groups including control, OVA - , and OVA + Bmab-treated by real-time PCR. 
The results showed that $I L-1 \beta$ and $T N F-\alpha$ expression was increased in treated animals with OVA $(p=0.004$, $p=0.0127$; respectively), compared to the control group; while treatment with Bmab (OVA + Bmab) caused a decrease in $I L-1 \beta$ and $T N F-\alpha$ mRNA levels that all were significant in comparison to OVA group $(P<0.05)$ (Fig. 3).

\section{Protein Expression Level of Cytokines in Lung Tissue}

To detect probable alterations in protein expression of pro-inflammatory factors, including TNF- $\alpha$ and IL- $1 \beta$ in asthma animal models (OVA group) and drug-treated group (OVA + Bmab) compared to the control group, immunohistochemistry assay was applied on lung tissue of all three groups. As shown in Fig. 4, the expression of IL-1 $\beta$ protein increased significantly in the OVA group $(p=0.02)$ compared to the control group. Parallel to IL-1 $\beta$ mRNA level, the Bmab treatment group showed a significant decrease similar to the TNF- $\alpha$ protein level $(p<0.0001)$ (Fig. 5).

\section{The Expression of VEGF Protein}

To evaluate the expression of VEGF protein in lung tissues and microgravity, IHC was performed. An obvious increase in the expression of VEGF protein in the OVA group was observed compared to the control group (Fig. 6; $p<0.011$ ). On the other hand, it was revealed that $\mathrm{Bmab}$ in the OVA group $(\mathrm{OVA}+\mathrm{Bmab}$ group) reduced the expression of VEGF protein $(p=0.01)$ compared to the OVA group (Fig. 6).

$\mathbf{a}$

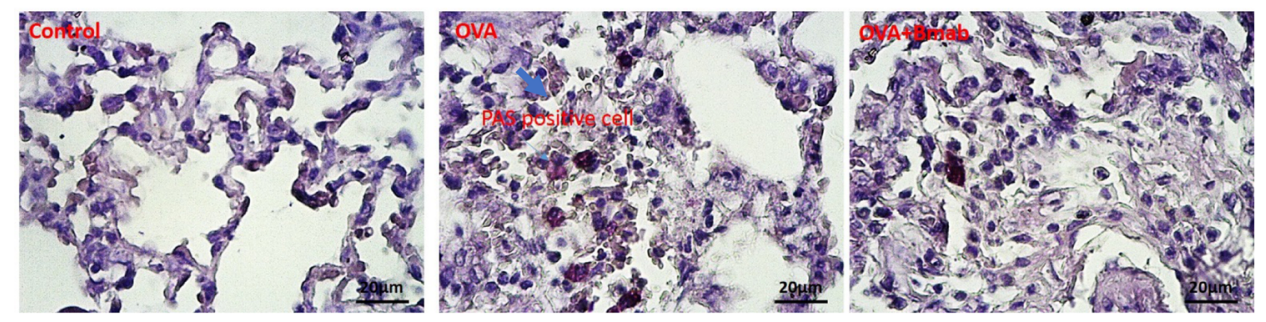

b

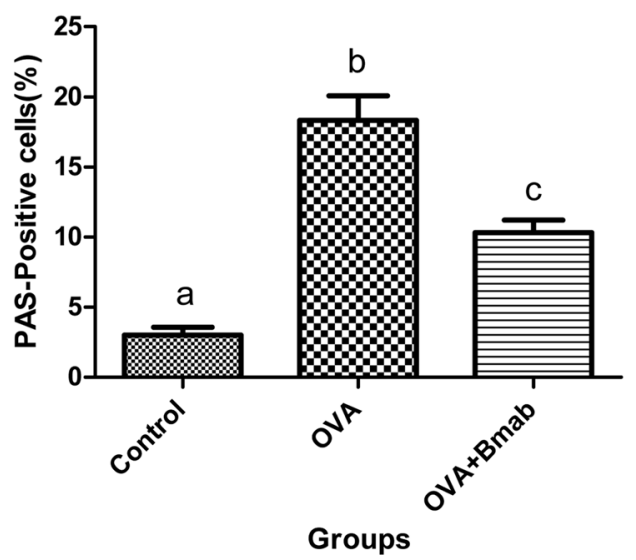

Fig. 2 Effects of bevacizumab on airway goblet cell hyperplasia by PAS staining of lung sections. A Goblet cells hyperplasia in OVA-induced rat model of asthma, magnification: $400 \times$. B Quantitated PAS-positive cells are illustrated as a graph. Different signs show significant differences between experimental groups. $p<0.01$ compared with control rats. PAS, periodic acid Schiff; OVA, ovalbumin; Bmab, bevacizumab. 


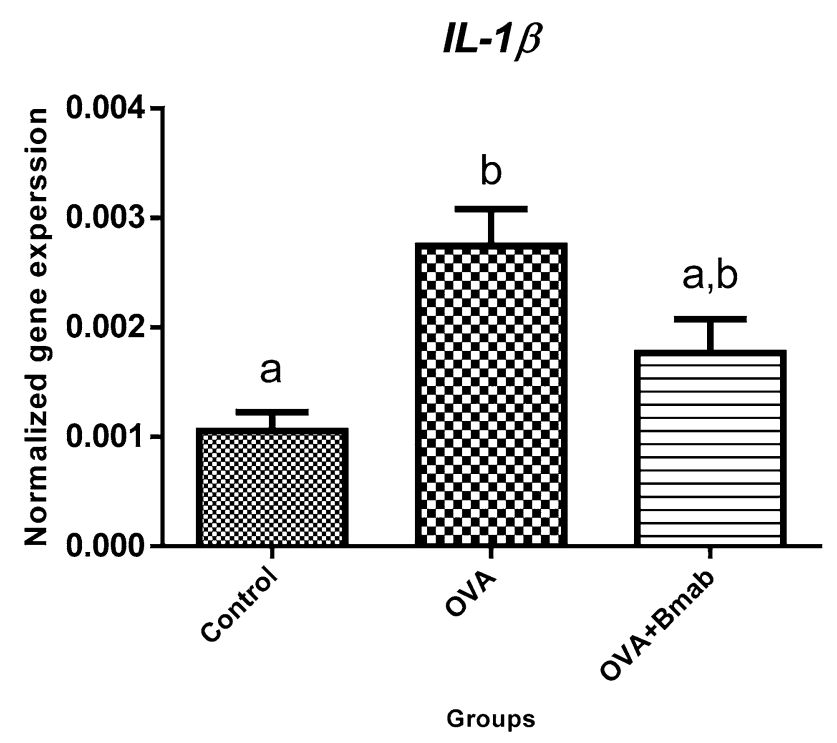

TNF- $\alpha$

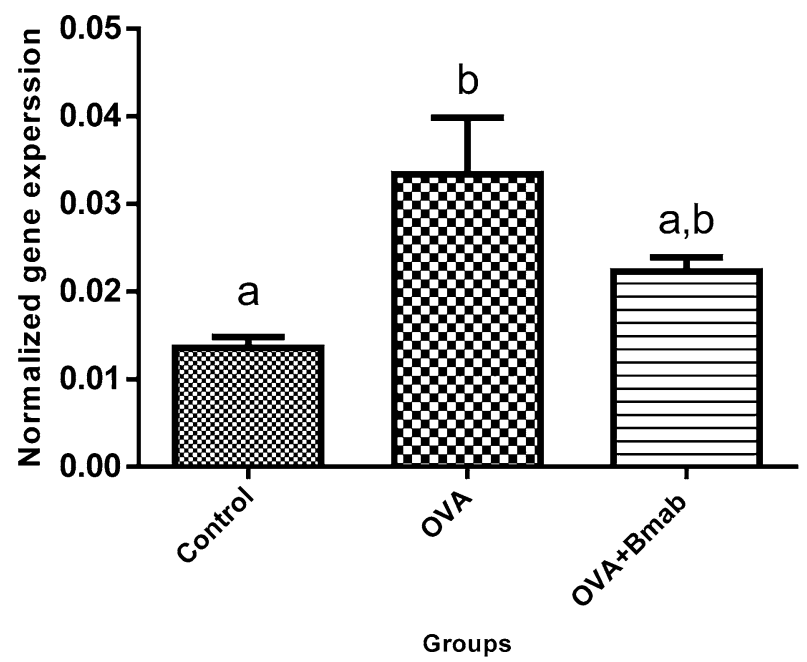

Fig. 3 Effects of bevacizumab on OVA-induced lung inflammatory genes expression. TNF- $\alpha$ and $I L-1 \beta$ changes were assessed by real-time PCR in experimental groups. Data are expressed as means \pm SE. Different signs represent statistically significant differences between the mean values $(p=0.004 ; I L-1 \beta, p=0.0127 ; T N F-\alpha)$ and similar signs are not significant. OVA, ovalbumin; Bmab, bevacizumab.

\section{Counting of BALF Leukocytes}

As shown in Fig. 7, Bmab treatment could significantly reduce the number of macrophages, neutrophils, and lymphocytes in lung tissue compared to the asthma group $(p<0.05)$, while no significant difference in the number of eosinophils was observed among the OVA and OVA + Bmab.

\section{Serum OVA-Specific IgE}

A higher level of OVA-specific IgE was detected in the asthma group compared to the control $(p<0.05)$. However, the results in treatment groups revealed that Bmab significantly suppressed OVA-specific IgE secretion in the treated group $(P<0.05)$ (Fig. 8). 

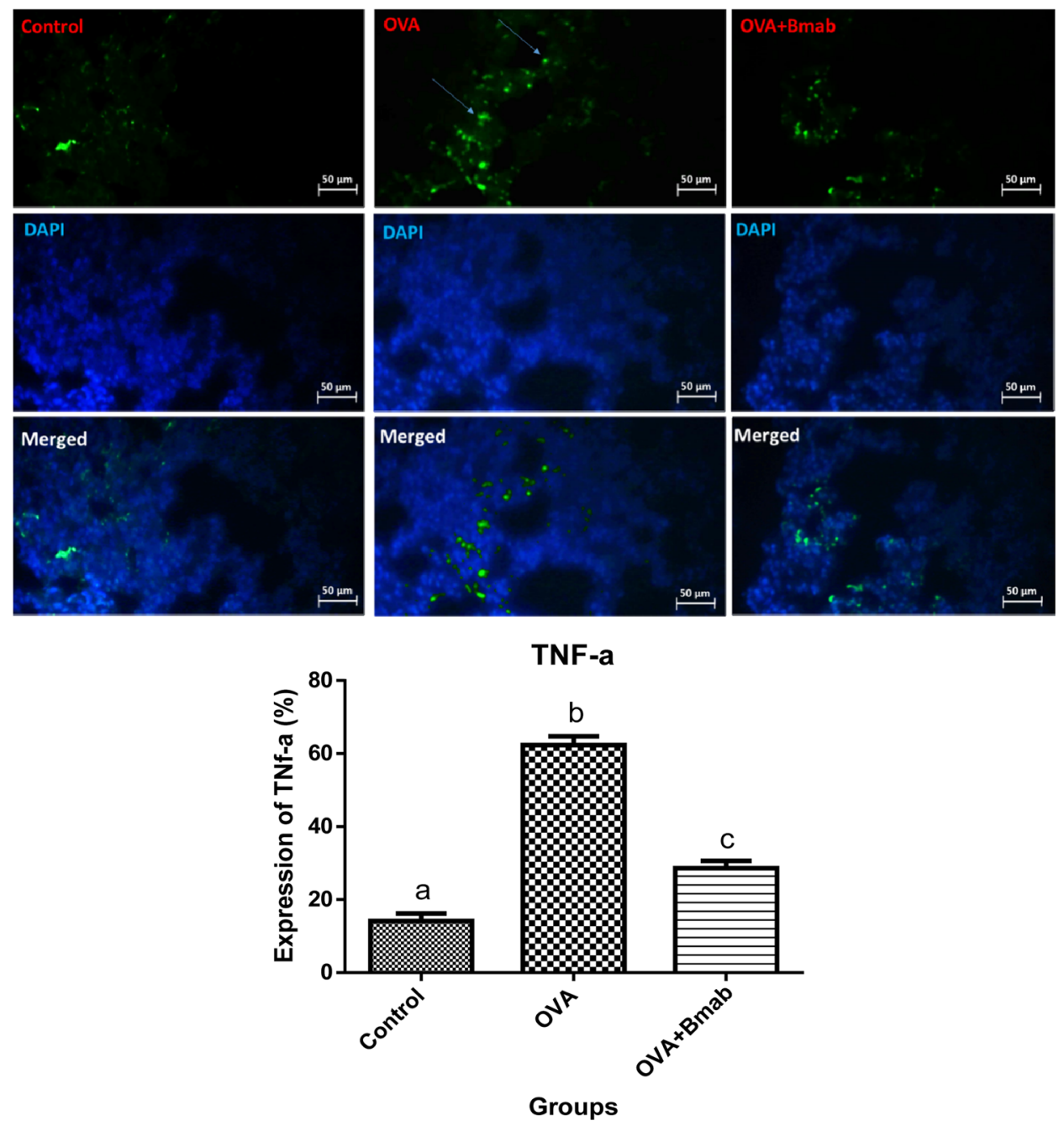

Fig. 4 Effects of bevacizumab on OVA-induced lung inflammatory protein of TNF- $\alpha$. Immunohistochemical staining was performed in rats' lung sections. Data are expressed as means \pm SE. Different signs represent statistically significant differences between the mean values $(p<0.02)$ and similar signs are not significant. OVA, ovalbumin; Bmab, bevacizumab.

\section{DISCUSSION}

In the present study, a new strategy was introduced to treat symptoms of asthma. Bevacizumab-treated animals displayed a decrease in goblet cell population and migration in airways of mice treated with OVA treatment, indicating symptoms of asthma. The asthmatic airway mucosa inflammation is usually associated with enhanced vascular permeability and plasma exudation. Vesicular-vascular organelles which are present largely in venular endothelium provide the major route of extravasation of macromolecules at sites of increased vascular permeability [34-37]. In the airways, the plasma exudation may readily pass through the inflamed mucosa and reach the airway lumen through the leaky epithelium. Leaked plasma proteins induce a thickened, engorged, and edematous airway wall, resulting in airway obstruction. The leaked plasma proteins can also traverse the epithelium and agglomerate in the airway lumen, leading to a reduction of mucus clearance and ciliary dysfunction [38]. Due to the interaction between VEGF and inflammation situation, our results showed that VEGF inhibition by bevacizumab might influence or possibly inhibit the inflammatory activity of inflammation cells in BALF. 

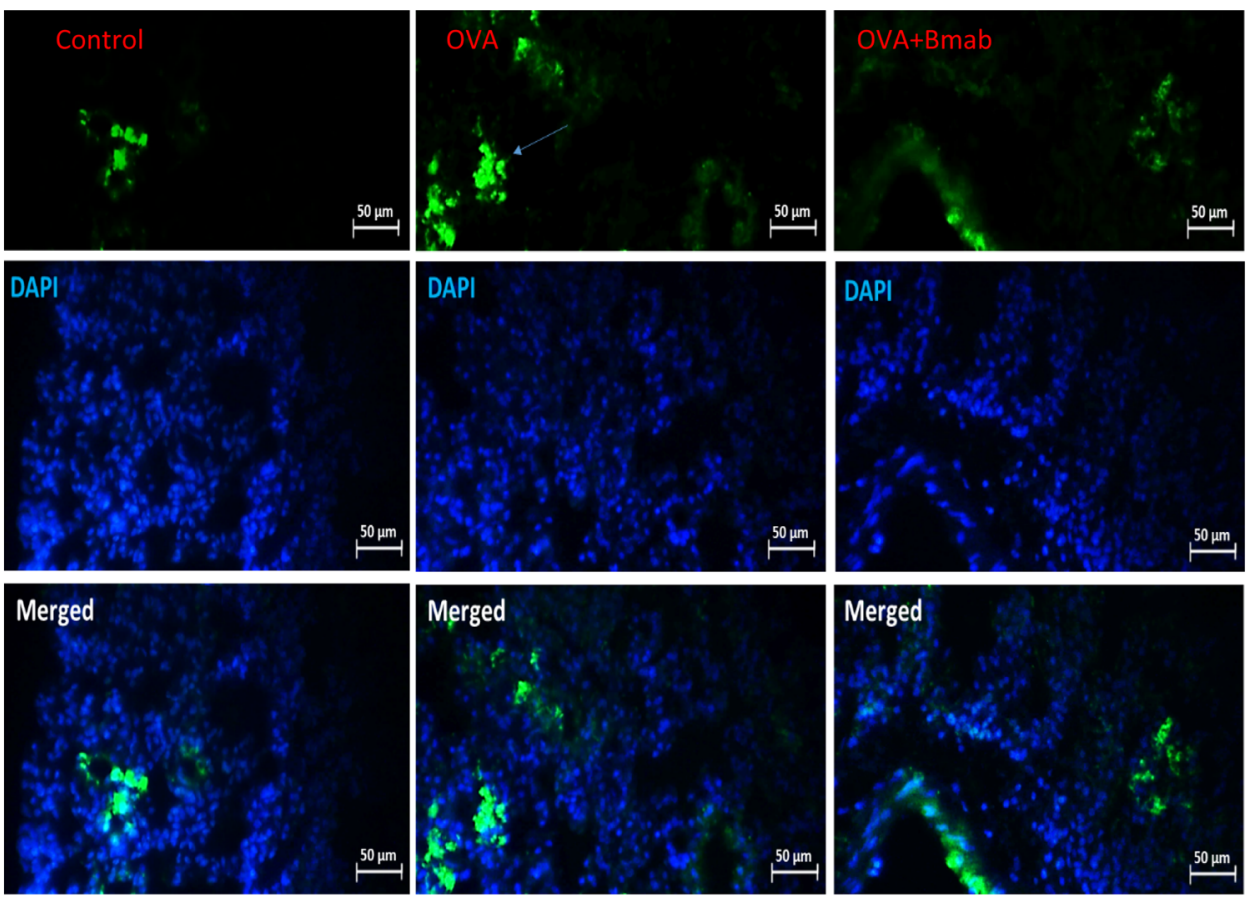

IL-1 $\beta$

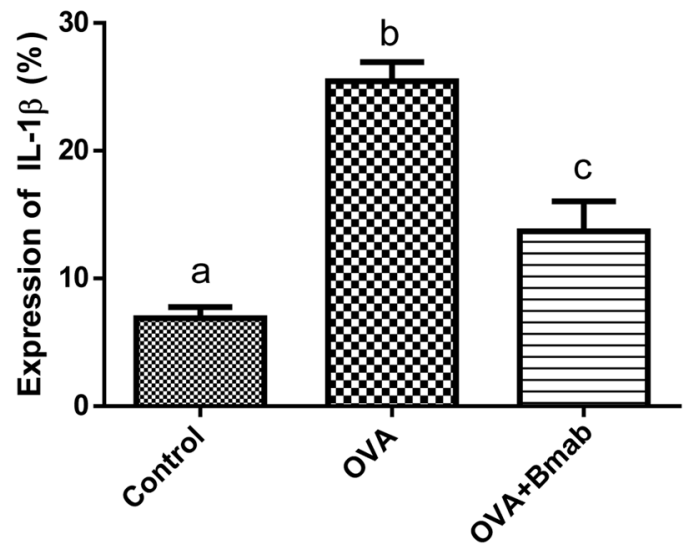

Groups

Fig. 5 Effects of bevacizumab on OVA-induced lung inflammatory protein of IL-1 $\beta$. Immunohistochemical staining was performed in rats' lung sections. Data are expressed as means \pm SE. Different signs represent statistically significant differences between the mean values $(p<0.0001)$ and similar signs are not significant. OVA, ovalbumin; Bmab, bevacizumab.

The density of leukocytes and especially of macrophages and neutrophils was lower in the OVA-bevacizumab treated group compared to the OVA group. Macrophages, in addition to stimulating VEGF, can induce epithelium of airway lumen proliferation and migration through the release of cytokines, proteases, and growth factors based on the results of Sunderkötter et al. in 1994 [39]. Therefore, experimental studies for potential new antiangiogenic treatment strategies target the complement system [40], ICAM [41], macrophages, monocytes [42, 43], and TNF [44]. Tsuchihashi S et al. in 2006 investigated that anti-VEGF drugs might be expected to have 

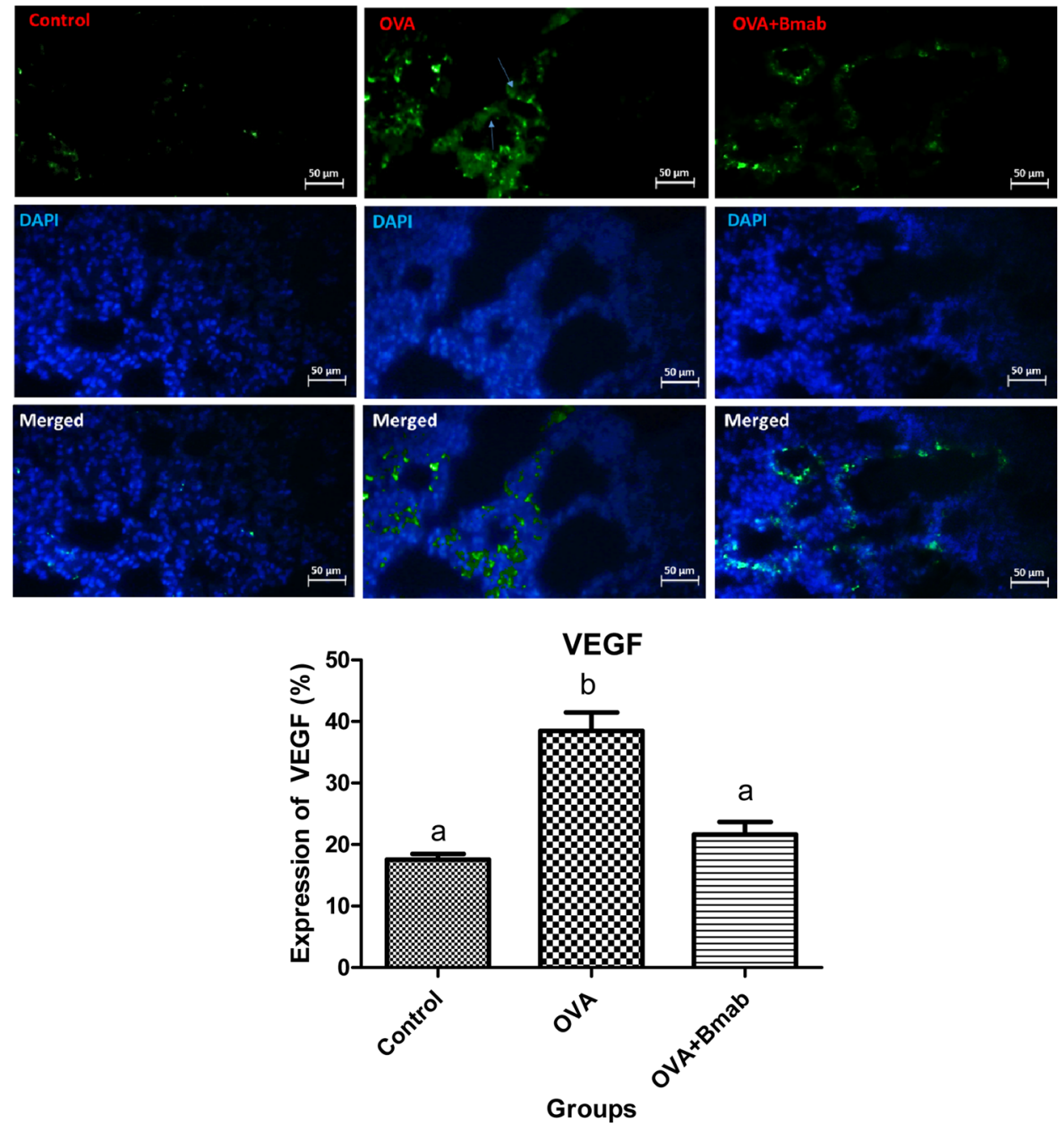

Fig. 6 Effects of bevacizumab on OVA-induced lung inflammatory protein of VEGF. Immunohistochemical staining was performed in rats' lung sections. Data are expressed as means \pm SE. Different signs represent statistically significant differences between the mean values $(p<0.01)$, and similar signs are not significant. OVA, ovalbumin; Bmab, bevacizumab.

an anti-inflammatory effect [45]. VEGF acts as a survival factor for epithelium growth of airway lumen by inhibiting apoptosis. Alon et al. revealed that deprivation and/or inhibition of VEGF could induce vascular regression through epithelial cell apoptosis in the retina, especially in neovascular vessels [46]. Therefore, the usage of bevacizumab as anti-angiogenesis drug was employed to prevent angiogenesis and reduce access to inflammatory cells in the lung tissue area. Microvascular leakage is an essential component of the inflammatory responses, and the majority of inflammatory mediators induce this vascular leakage in asthma [47-50]. On the basis of COVID-19-induced pulmonary pathological and vascular changes, it can be hypothesized that the antivascular endothelial growth factor (VEGF) drug bevacizumab plus standard care might be highly beneficial for patients with severe COVID-19 [51]. The results of the present study showed that VEGF expression decreased in the group receiving bevacizumab compared to that of the asthma group. VEGF by binding to VEGF triggers a signaling network in endothelial cells which causes angiogenesis [52]. Thus, VEGF is an endothelial marker and its reduction demonstrated that bevacizumab can reduce the rate of angiogenesis in lung tissue. In the 


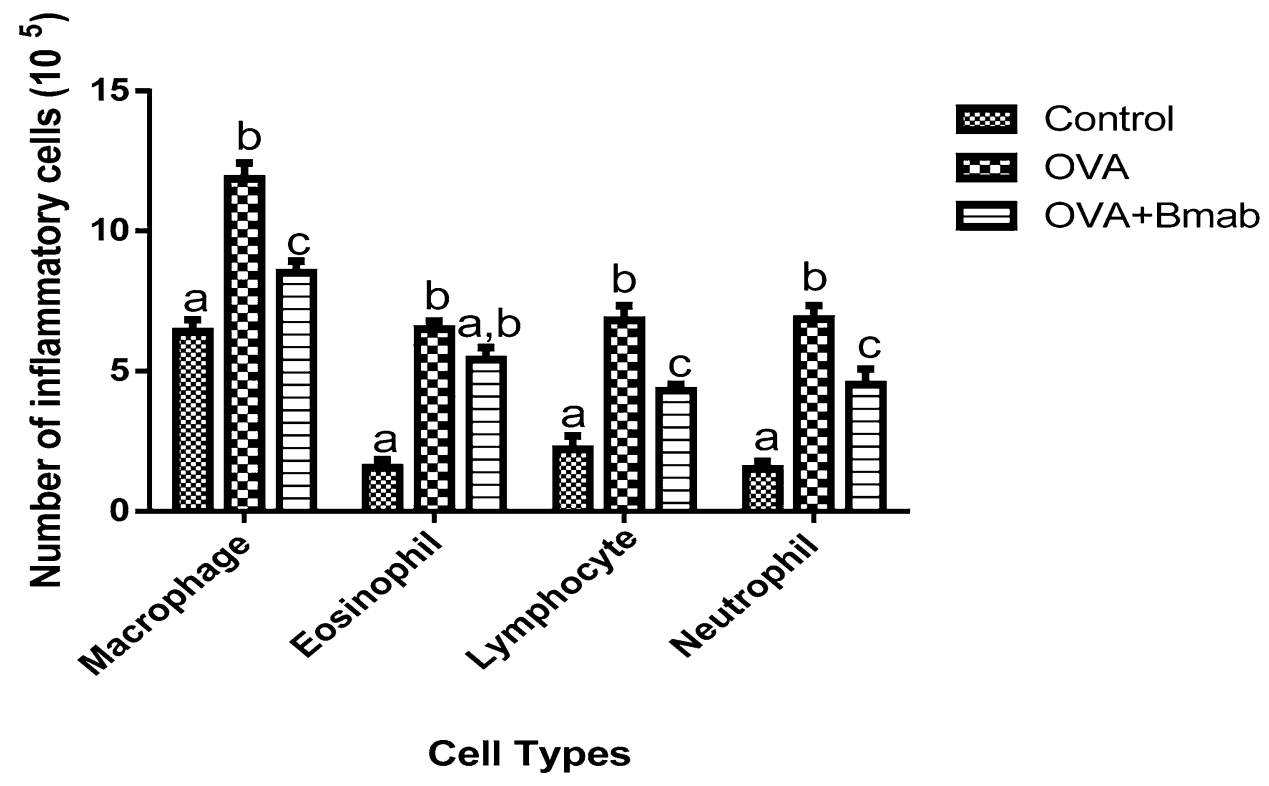

Fig. 7 The number of inflammatory cells in bronchoalveolar lavage fluid (BALF) of the experimental groups. Data are expressed as means \pm SE. Different signs represent statistically significant differences between the mean values $(p<0.01)$, and similar signs are not significant. OVA, ovalbu$\mathrm{min}$; Bmab, bevacizumab.

case of wound healing, VEGF expression, as one of the vital angiogenic stimulators, is significantly upregulated to accelerate wound healing by regulating and restoring blood flow to the injured area. As wound healing resolves, the expression of VEGF is downregulated, and most angiogenic capillaries regress, resulting in residual normal vascularity [53]. New evidence indicates that a number of endogenous angiogenic inhibitors are

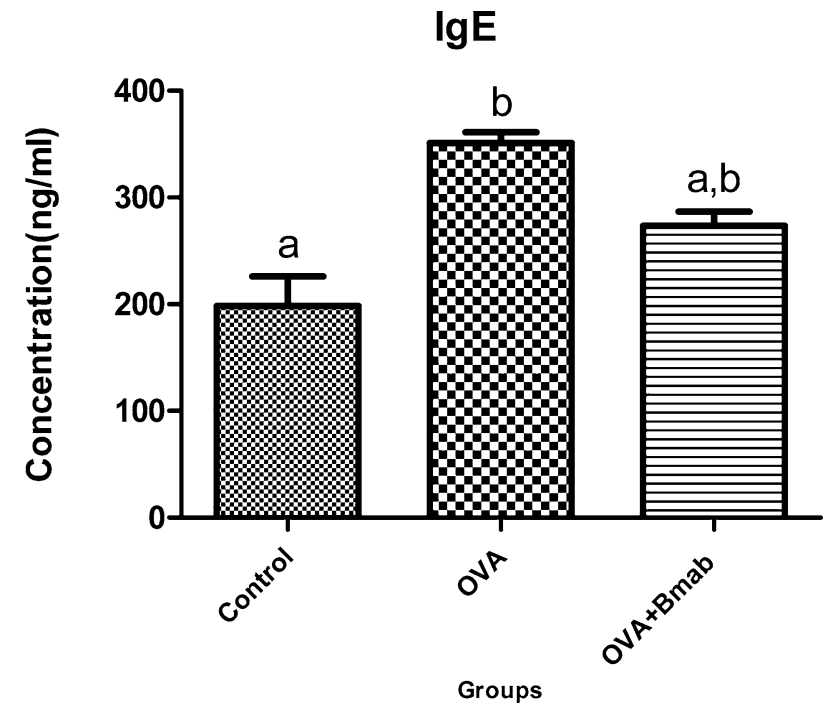

Fig. 8 Serum OVA-IgE concentration in control, OVA-sensitized and OVA-bevacizumab treatment. Data are expressed as means \pm SE. Different signs represent statistically significant differences between the mean values $(p<0.01)$ and similar signs are not significant. OVA, ovalbumin; Bmab, bevacizumab. 
present in the normal retina to balance the stimulatory effect of VEGF in the regulation of angiogenesis and vascular permeability [54]. It is suggested that endogenous angiogenic inhibitors can be utilized to balance the effect of angiogenic stimulators. TNF- $\alpha$ and IL- $1 \beta$ were known as major regulatory cytokines which can initiate the inflammatory. These processes are performed by switching on many downstream genes, inducing expression of signaling molecules and transcription factors, cytokines, chemokines, components of the coagulation system, cell surface receptors and adhesion molecules, cell proliferation-related genes, apoptosis-related genes, and other inflammatory response genes $[30,55,56]$. Our study showed that the expression of TNF- $\alpha$ and IL- $1 \beta$ increased in the induced rat model of asthma, but their expression decreased significantly in the asthmatic animal group receiving bevacizumab compared with the non-treated group; however, their expression is higher than the control group. Hence, bevacizumab can have good effects on inflammatory diseases such as asthma. Some studies have reported the increase of TNF- $\alpha$ and lymphotoxin alpha expression in mouse lungs after $\mathrm{M}$. pulmonic infection [57-60], but in the viewpoint of vessel remodeling in the airways, no report has been published yet. Lung expression of TNF- $\alpha$ increases within a few hours of infection and then decreases to near baseline values [57-59], but TNF- $\alpha$ expression is stable for a period of time in the trachea. This difference may indicate a histological difference in the cell population in the trachea and lung, as well as a complex combination of parenchymal alveoli and intrapulmonary airways. Evaluation of the relationship between TNF- $\alpha$ expression and vascular regeneration or lymphatic angiogenesis in the trachea indicates an increase in TNF- $\alpha$ involvement in vascular regeneration. This therefore suggests that bevacizumab controls asthma by angiogenesis inhibition through the reduction of TNF- $\alpha$ and IL- $1 \beta$ expression. Decreased expression of chemoattractants and adhesive molecules may help reduce the loss of leukocytes and lymph node hypertrophy in infected mice treated with anti-TNF antibodies or TNF-R1 deficiency. Similarly, TNF- $\alpha$ causes the expression of VCAM-1, E-selectin, and ICAM-1 in endothelial cells, which mediates the adhesion and exit of leukocytes [61]. Among the logical approaches for airway diseases such as severe asthma and chronic obstructive pulmonary, blocking TNF- $\alpha$ signaling would seem to be a noticeable therapy [62]. Recent clinical studies of asthma treated with anti-TNF- $\alpha$ have improved lung function and quality of life and reduced intensified airway responses [63-65]. Therefore, this type of therapy may need to be administered over a long period of time. In the normal healthy body, the process of angiogenesis is dormant, and the angiogenesis switch is kept "off" with inhibitors that are dominant over stimulators. Since anti-angiogenesis therapy is a targeted therapy that aims specifically at the angiogenic stimulators and the angiogenic microvascular endothelial cells, anti-angiogenesis therapy usually produces only mild side effects and is less toxic to most healthy cells. Meanwhile, some investigations have shown that intravitreal injection of bevacizumab may induce a significant rise in IOP [14]. It is important to mention that since angiogenesis is vital in the wound healing process and reproduction, anti-angiogenic agents in long-term treatments can result in problems in case of bleeding, heart function, blood clotting, the immune system problems, and the reproductive system, which are still unknown and need to be studied $[66,67]$

\section{CONCLUSION}

Based on the present investigation, angiogenesis plays an important role in the expression and release of inflammatory mediators in lung airways in asthma. Targeting VEGF through the application of bevacizumab would be a great therapeutic option to prevent the complication of asthma.

\section{AUTHOR CONTRIBUTIONS}

Seyed Mohammadreza Bolandi, Zohreh Abdolmaleki, and Mohammad-Ali Assarehzadegan searched the literature and designed the study. All the authors performed the experiments, analyzed the data, and wrote the manuscript. Seyed Mohammadreza Bolandi and Zohreh Abdolmaleki cheng revised the manuscript.

\section{Declarations}

Ethics Approval The study procedures were performed according to the Ethical Committee of Azad University. The ethical code assigned for this study was IR.IAU.K.REC.1398.088.

Conflict of Interest The authors declare no competing interests. 


\section{REFERENCES}

1. Jones, A.W., and R.E. Gemine. 2021. Patients living with other respiratory diseases. Supporting Tobacco Cessation 90: 193.

2. Peredo, R.A., V. Mehta, and S. Beegle. 2021. Interstitial lung disease associated with connective tissue diseases. In Lung inflammation in health and disease, vol. II, 73-94. Springer.

3. Handoko, D., F. Yunus, and B. Antariksa. 2021. Osteoporosis prevalence in stable patients with chronic obstructive pulmonary disease. Respiratory Science 1 (2): 132-149.

4. Roofeh, D., et al. 2021. Tocilizumab prevents progression of early systemic sclerosis associated interstitial lung disease. Arthritis \& Rheumatology, 73 (7):1301-1310

5. Diller, G.-P., et al. 2021. Coronavirus disease 2019 in adults with congenital heart disease: A position paper from the ESC working group of adult congenital heart disease, and the International Society for Adult Congenital Heart Disease. European Heart Journal 42 (19): 1858-1865.

6. Diller, G.-P., and M.A. Gatzoulis. 2007. Pulmonary vascular disease in adults with congenital heart disease. Circulation 115 (8): 1039-1050.

7. Mirdar, S., et al. 2019. The effects of tapering with and without ethanolic extract of Nigella sativa on Hypoxia Inducible Factor- $1 \alpha$ and exercise-induced bronchial changes. Journal of Military Medicine 21 (2): 131-141.

8. Park, H.S., et al. 2010. Targeting abnormal airway vascularity as a therapeutical strategy in asthma. Respirology 15 (3): 459-471.

9. Tagaya, E., and J. Tamaoki. 2007. Mechanisms of airway remodeling in asthma. Allergology International 56 (4): 331-340.

10. Hu, L., et al. 2019. Inhibition of airway remodeling and inflammatory response by icariin in asthma. BMC Complementary and Alternative Medicine 19 (1): 1-10.

11. Shojaei, F. 2012. Anti-angiogenesis therapy in cancer: Current challenges and future perspectives. Cancer Letters 320 (2): $130-137$.

12. Grothey, A., and E. Galanis. 2009. Targeting angiogenesis: Progress with anti-VEGF treatment with large molecules. Nature Reviews Clinical Oncology 6 (9): 507.

13. Huang, C., et al. 2016. Bevacizumab reduced auto-phosphorylation of VEGFR2 to protect HDM-induced asthma mice. Biochemical and Biophysical Research Communications 478 (1): 181-186.

14. Lemos-Reis, R., et al. 2014. Immediate effect of intravitreal injection of bevacizumab on intraocular pressure. Clinical Ophthalmology (Auckland, N.Z.) 8: 1383-1388.

15. Peak, S.J., and V.A. Levin. 2010. Role of bevacizumab therapy in the management of glioblastoma. Cancer Management and Research 2: 97-104.

16. Chand, N., et al. 1992. Anti-IL-5 monoclonal antibody inhibits allergic late phase bronchial eosinophilia in guinea pigs: A therapeutic approach. European Journal of Pharmacology 211 (1): $121-123$.

17. Lugano, R., M. Ramachandran, and A. Dimberg. 2020. Tumor angiogenesis: Causes, consequences, challenges and opportunities. Cellular and Molecular Life Sciences 77 (9): 1745-1770.

18. Samitas, K., et al. 2018. Upper and lower airway remodelling mechanisms in asthma, allergic rhinitis and chronic rhinosinusitis: The one airway concept revisited. Allergy 73 (5): 993-1002.

19. Csete, M.E., W.M. Abraham, and A. Wanner. 1990. Vasomotion Influences Airflow in Peripheral Airways1-3. The American Review of Respiratory Disease 141: 1409-1413.
20. Blosser, S., W. Mitzner, and E.M. Wagner. 1994. Effects of increased bronchial blood flow on airway morphometry, resistance, and reactivity. Journal of Applied Physiology 76 (4): 1624-1629.

21. Zheng, F., et al. 2017. Epinephrine but not vasopressin attenuates the airway response to anaphylactic shock in rats. Experimental Lung Research 43 (3): 158-166.

22. Long, W., et al. 1988. Modification of bronchial blood flow during allergic airway responses. Journal of Applied Physiology 65 (1): 272-282.

23. Wanner, A. 1989. Circulation of the airway mucosa. Journal of Applied Physiology 67 (3): 917-925.

24. Webber, S., et al. 1990. Effects of non-neural mediators and allergen on tracheobronchial blood flow. The European Respiratory Journal. Supplement 12: 638s-643s.

25. Pua, H., H., et al. 2019. Increased hematopoietic extracellular RNAs and vesicles in the lung during allergic airway responses. Cell Reports 26 (4): 933-944.

26. Lumb, A., B. and C., R. Thomas. 2020. Nunn's applied respiratory physiology eBook. Elsevier Health Sciences.

27. Barnes, P.J., and J.M. Drazen. 2002. Pathophysiology of asthma. In Asthma and COPD, 343-359. Elsevier.

28. Hikichi, M., S. Hashimoto, and Y. Gon. 2018. Asthma and COPD overlap pathophysiology of ACO. Allergology International 67 (2): 179-186.

29. Chetta, A., et al. 2003. Vascular component of airway remodeling in asthma is reduced by high dose of fluticasone. American Journal of Respiratory and Critical Care Medicine 167 (5): 751-757.

30. Gallagher, S.J., et al. 2002. Methods to track leukocyte and erythrocyte transit through the bronchial vasculature in sheep. Journal of Immunological Methods 271 (1-2): 89-97.

31. She, W., et al. 2019. Nebulized inhalation of anti-nerve growth factor microspheres inhibits airway remodeling in an ovalbumininduced rat asthma model. Journal of Aerosol Medicine and Pulmonary Drug Delivery 32 (2): 70-77.

32. Soysal, D., et al. 2014. A novel angiogenesis inhibitor bevacizumab induces apoptosis in the rat endometriosis model. Balkan Journal of Medical Genetics 17 (2): 73-80.

33. Yang, Y.G., et al. 2013. Nerve growth factor exacerbates allergic lung inflammation and airway remodeling in a rat model of chronic asthma. Experimental and Therapeutic Medicine 6 (5): 1251-1258.

34. Dvorak, H.F., et al. 1999. Vascular permeability factor/vascular endothelial growth factor and the significance of microvascular hyperpermeability in angiogenesis. In Vascular growth factors and angiogenesis, 97-132. Springer.

35. Nagy, J.A., et al. 2008. Vascular permeability, vascular hyperpermeability and angiogenesis. Angiogenesis 11 (2): 109-119.

36. Nag, S., et al. 2019. Increased expression of vascular endothelial growth factor-D following brain injury. International Journal of Molecular Sciences 20 (7): 1594.

37. Probst, C.K., et al. 2020. Vascular permeability in the fibrotic lung. European Respiratory Journal 56 (1): 1900100.

38. Foster, W. M., Langenback, E. G., and E., H. Bergofsky. 1982. Lung mucociliary function in man: interdependence of bronchial and tracheal mucus transport velocities with lung clearance in bronchial asthma and healthy subjects. In Inhaled Particles 2(7): 227-244.

39. Sunderkötter, C., et al. 1994. Macrophages and angiogenesis. Journal of Leukocyte Biology 55 (3): 410-422.

40. Bora, N.S., et al. 2007. CD59, a complement regulatory protein, controls choroidal neovascularization in a mouse model of wettype age-related macular degeneration. The Journal of Immunology 178 (3): 1783-1790. 
41. Sakurai, E., et al. 2003. Targeted disruption of the CD18 or ICAM-1 gene inhibits choroidal neovascularization. Investigative Ophthalmology \& Visual Science 44 (6): 2743-2749.

42. Sakurai, E., et al. 2003. Macrophage depletion inhibits experimental choroidal neovascularization. Investigative Ophthalmology \& Visual Science 44 (8): 3578-3585.

43. Espinosa-Heidmann, D.G., et al. 2003. Macrophage depletion diminishes lesion size and severity in experimental choroidal neovascularization. Investigative Ophthalmology \& Visual Science 44 (8): 3586-3592.

44. Krzystolik, M.G., et al. 2002. Prevention of experimental choroidal neovascularization with intravitreal anti-vascular endothelial growth factor antibody fragment. Archives of Ophthalmology 120 (3): 338-346.

45. Tsuchihashi, S.-I., et al. 2006. Vascular endothelial growth factor antagonist modulates leukocyte trafficking and protects mouse livers against ischemia/reperfusion injury. The American Journal of Pathology 168 (2): 695-705.

46. Alon, T., et al. 1995. Vascular endothelial growth factor acts as a survival factor for newly formed retinal vessels and has implications for retinopathy of prematurity. Nature Medicine 1 (10): 1024-1028.

47. Kanazawa, H., et al. 2002. Vascular involvement in exerciseinduced airway narrowing in patients with bronchial asthma. Chest 122 (1): 166-170.

48. Lim, L.H., B.S. Bochner, and E.M. Wagner. 2002. Leukocyte recruitment in the airways: An intravital microscopic study of rat tracheal microcirculation. American Journal of Physiology-Lung Cellular and Molecular Physiology 282 (5): L959-L967.

49. Kelly, L., et al. 1986. Bronchial blood flow affects recovery from constriction in dog lung periphery. Journal of Applied Physiology 60 (6): 1954-1959.

50. Csete, M. E., Chediak, A. D., Abraham, W. M., \& Wanner, A. 1991. Airway Blood Flow Modifies Allergic Airway Smooth Muscle Contraction, American Review of Respiratory Disease 144(1) 1-3.

51. Pang, J., et al. 2021. Efficacy and tolerability of bevacizumab in patients with severe Covid-19. Nature Communications 12 (1): 814.

52. Folkman, J. 1974. Proceedings: Tumor angiogenesis factor. Cancer Research 34 (8): 2109-2113.

53. Huh, J.E., et al. 2009. Formononetin promotes early fracture healing through stimulating angiogenesis by up-regulating VEGFR-2/Flk-1 in a rat fracture model. International Immunopharmacology 9 (12): $1357-1365$.

54. Nocker, R.E., et al. 1999. A double-blind study on the effect of inhaled corticosteroids on plasma protein exudation in asthma. American Journal of Respiratory and Critical Care Medicine 159 (5): 1499-1505.

55. Barbato, A., et al. 2006. Epithelial damage and angiogenesis in the airways of children with asthma. American Journal of Respiratory and Critical Care Medicine 174 (9): 975-981.
56. Mitzner, W., and E.M. Wagner. 2004. Vascular remodeling in the circulations of the lung. Journal of Applied Physiology 97 (5): 1999-2004.

57. Faulkner, C.B., et al. 1995. Gene expression and production of tumor necrosis factor alpha, interleukin 1 , interleukin 6 , and gamma interferon in $\mathrm{C} 3 \mathrm{H} / \mathrm{HeN}$ and C57BL/6N mice in acute Mycoplasma pulmonis disease. Infection and Immunity 63 (10): 4084-4090.

58. Lewis, C.C., et al. 2008. Disease-specific gene expression profiling in multiple models of lung disease. American Journal of Respiratory and Critical Care Medicine 177 (4): 376-387.

59. Sun, X., et al. 2006. Cytokine and chemokine transcription profile during Mycoplasma pulmonis infection in susceptible and resistant strains of mice: Macrophage inflammatory protein $1 \beta$ (CCL4) and monocyte chemoattractant protein 2 (CCL8) and accumulation of CCR5+ Th cells. Infection and Immunity 74 (10): 5943-5954.

60. Tayebi, S.M., et al. 2019. Plasma retinol-binding protein-4 and tumor necrosis factor- $\alpha$ are reduced in postmenopausal women after combination of different intensities of circuit resistance training and Zataria supplementation. Sport Sciences for Health 15 (3): 551-558.

61. Viemann, D., et al. 2004. Transcriptional profiling of IKK2/ $\mathrm{NF}-\mathrm{KB}$ - and p38 MAP kinasedependent gene expression in TNF- $\alpha$ - stimulated primary human endothelial cells. Blood 103 (9): 3365-3373.

62. Barnes, P.J. 2003. Cytokine-directed therapies for the treatment of chronic airway diseases. Cytokine \& Growth Factor Reviews 14 (6): 511-522

63. Berry, M., et al. 2007. TNF- $\alpha$ in asthma. Current Opinion in Pharmacology 7 (3): 279-282.

64. Berry, M.A., et al. 2006. Evidence of a role of tumor necrosis factor $\alpha$ in refractory asthma. New England Journal of Medicine 354 (7): 697-708.

65. Erin, E.M., et al. 2006. The effects of a monoclonal antibody directed against tumor necrosis factor- $\alpha$ in asthma. American Journal of Respiratory and Critical Care Medicine 174 (7): 753-762.

66. Helisch, A., et al. 2006. Impact of mouse strain differences in innate hindlimb collateral vasculature. Arteriosclerosis, Thrombosis, and Vascular Biology 26 (3): 520-526.

67. Heil, M., et al. 2006. Arteriogenesis versus angiogenesis: Similarities and differences. Journal of Cellular and Molecular Medicine 10 (1): 45-55.

Publisher's Note Springer Nature remains neutral with regard to jurisdictional claims in published maps and institutional affiliations. 